\title{
Simple non-invasive assessment of advanced glycation endproduct accumulation
}

\author{
R. Meerwaldt ${ }^{1}$ R. Graaff ${ }^{2}$ P. H. N. Oomen ${ }^{1}$ T. T. Links ${ }^{1}$ J. J. Jager ${ }^{3}$ • N. L. Alderson ${ }^{4}$ S. R. Thorpe • $^{4}$ \\ J. W. Baynes ${ }^{4}$ R. O. B. Gans ${ }^{1}$ - A. J. Smit ${ }^{1}$ \\ ${ }^{1}$ Divisions of Vascular Medicine and Endocrinology, Department of Medicine, University Hospital Groningen, Groningen, \\ The Netherlands \\ 2 Department of Biomedical Engineering, University of Groningen, Groningen, The Netherlands \\ ${ }^{3}$ Department of Medicine, Diaconessen Hospital, Meppel, The Netherlands \\ ${ }^{4}$ Department of Chemistry and Biochemistry, University of South Carolina, Columbia, South Carolina, USA
}

\section{Abstract}

Aims/hypothesis. The accumulation of AGE is thought to play a role in the pathogenesis of chronic complications of diabetes mellitus and renal failure. All current measurements of AGE accumulation require invasive sampling. We exploited the fact that several AGE exhibit autofluorescence to develop a non-invasive tool for measuring skin AGE accumulation, the Autofluorescence Reader (AFR). We validated its use by comparing the values obtained using the AFR with the AGE content measured in extracts from skin biopsies of diabetic and control subjects.

Methods. Using the AFR with an excitation light source of 300-420 nm, fluorescence of the skin was measured at the arm and lower leg in 46 patients with diabetes (Type 1 and 2) and in 46 age- and sexmatched control subjects, the majority of whom were Caucasian. Autofluorescence was defined as the average fluorescence per $\mathrm{nm}$ over the entire emission spectrum (420-600 nm) as ratio of the average fluorescence per $\mathrm{nm}$ over the $300-420-\mathrm{nm}$ range. Skin biopsies were obtained from the same site of the arm, and analysed for collagen-linked fluorescence (CLF)

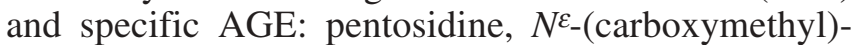
lysine (CML) and $N^{\varepsilon}$-(carboxyethyl)lysine (CEL).

Results. Autofluorescence correlated with CLF, pentosidine, CML, and CEL $(r=0.47-0.62, p \leq 0.002)$. In 32 of 46 diabetic patients (70\%), autofluorescence values were above the $95 \% \mathrm{CI}$ of the mean value in control subjects, and correlated with age, diabetes duration, mean $\mathrm{HbA}_{1} \mathrm{c}$ of the previous year and creatinine levels.

Conclusions/interpretation. The AFR offers a simple alternative to invasive measurement of AGE accumulation and, to date, has been validated in non-pigmented skin. The AFR may prove to be a useful clinical tool for rapid risk assessment of AGE-related long-term complications in diabetes mellitus and in other conditions associated with AGE accumulation.

Keywords AGE - Autofluorescence ·

Carboxyethyl-lysine $\cdot$ Carboxymethyl-lysine .

Non-invasive $\cdot$ Pentosidine
Received: 16 February 2004 / Accepted: 26 April 2004

Published online: 9 July 2004

C Springer-Verlag 2004

\author{
A. J. Smit ( $)$ \\ Divisions of Vascular Medicine and Endocrinology, \\ Department of Medicine U3.129, \\ University Hospital Groningen, Hanzeplein, \\ 9700 RB Groningen, The Netherlands \\ E-mail: a.j.smit@int.azg.nl \\ Tel.: +31-503616161, Fax: +31-503619069
}

Abbreviations: AFR, Autofluorescence Reader - CEL, $N^{\varepsilon}$-(carboxyethyl)lysine $\cdot$ CLF, collagen-linked fluorescence .

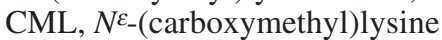

\section{Introduction}

The accumulation of AGE on tissue proteins has been implicated in the ageing of proteins and the progression of chronic, age-related diseases [1], such as atherosclerosis [2], chronic renal failure [3], Alzheimer's disease [4] and diabetes mellitus [5, 6, 7, 8]. Glucose initially reacts with proteins to form reversible early glycation products. AGE develop through complex rearrangements of these glycation products [1], and their formation is accelerated by decreased renal clearance of these products [3] and/or oxidative stress [9, 10]. The formation and accumulation of AGE on long- 

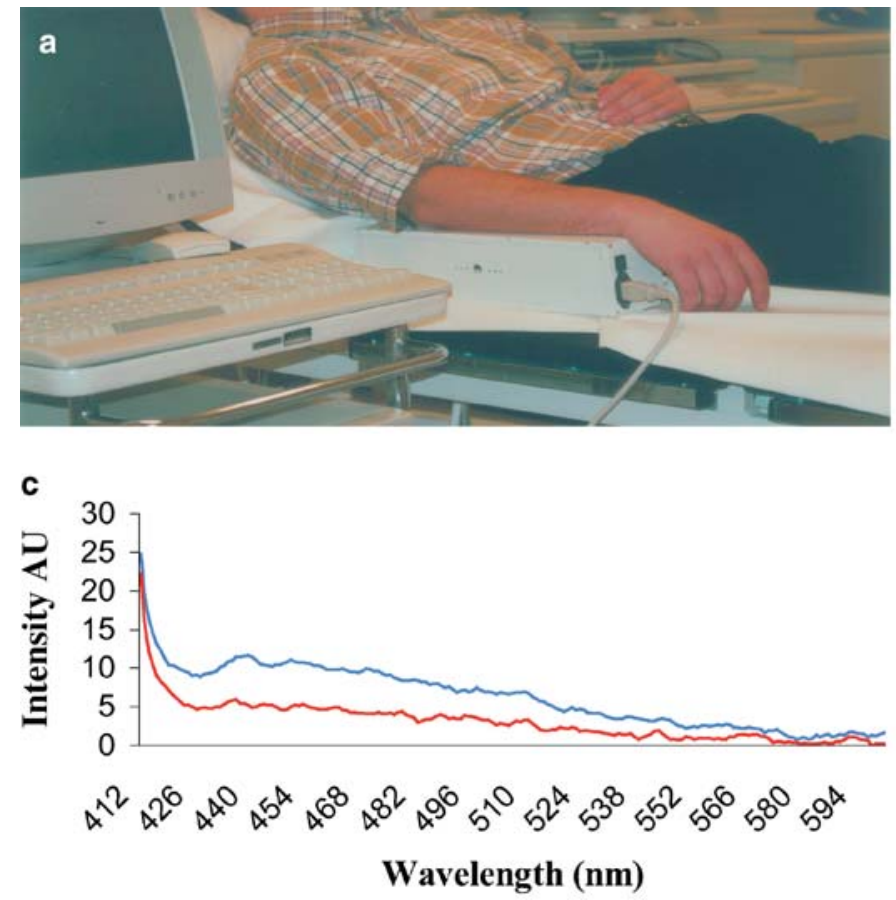

lived proteins affects the structure and function of proteins, enhances cytokine production and activates transcription factors via binding to specific receptors (e.g. the receptor for AGE) [11].

In diabetes mellitus, AGE accumulation in skin collagen is correlated both with the duration and severity of hyperglycaemia, and with the presence of long-term complications $[6,7,8,12,13,14,15]$. In a DCCT substudy, skin AGE levels explained 19 to $36 \%$ of the variance in the incidence of long-term diabetic complications in intensively treated patients, and 14 to $51 \%$ in conventionally treated patients [8]. These associations remained after adjustment for $\mathrm{HbA}_{1}$ c. Experimentally, prevention of AGE accumulation has been shown to reduce the development of several diabetic complications [16, 17, 18].

Upon excitation at $370 \mathrm{~nm}$, AGE have a characteristic fluorescence spectrum at $440 \mathrm{~nm}$. Classically, this has been used to determine tissue AGE accumulation, e.g. in extracts from homogenates of skin biopsies [5]. More recently developed biochemical and immunochemical assays measure both fluorescent AGE (e.g. pentosidine) and non-fluorescent AGE (e.g.

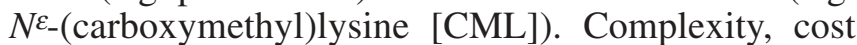
and lack of reproducibility limit the broader use of these later assays. Moreover, blood and urine sampling of AGE do not necessarily reflect tissue AGE levels $[19,20]$. Obviously, simple quantitation of AGE accumulation in tissue could provide a tool for assessing tissue injury and the risk of chronic complications. Using non-invasive fluorescence capillary microscopy, we noted increased skin autofluorescence in diabetic patients [21]. Because autofluorescence in other tissue (cornea, lens) is associated with the presence of AGE and diabetic retinopathy [22, 23], we de-

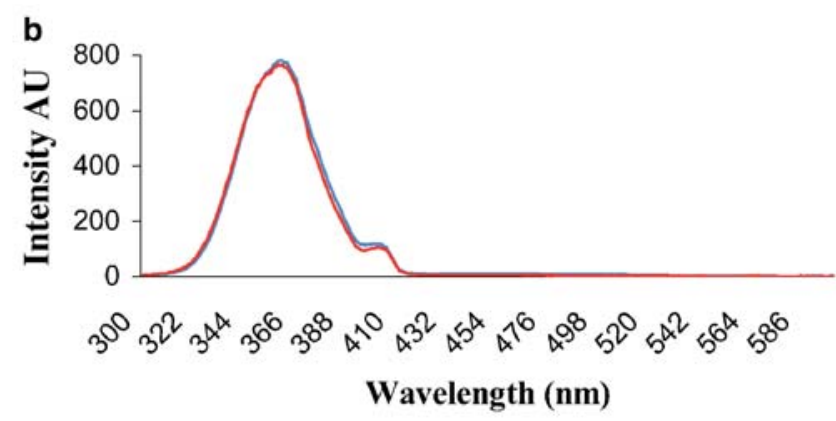

Fig. 1. a. Photograph of the Autofluorescence Reader (AFR). b. Light intensities emitted from the skin as analysed by the spectrometer across the 300-600-nm range for a Type 1 diabetic patient (blue line) and a matched control subject (red line). c. Finer detail of the emission spectrum (420-600 nm) for a Type 1 diabetic patient (blue line) and a matched control subject (red line). Definition of autofluorescence, see "Skin autofluorescence" (Subjects and methods)

veloped an instrument, the Autofluorescence Reader (AFR), that measures skin autofluorescence non-invasively.

The aims of the present study were to validate the AFR as a tool for the non-invasive assessment of AGE accumulation in skin by comparing measurements with those obtained using AGE assays in skin biopsies, and to evaluate the AFR's first application in diabetes.

\section{Subjects and methods}

Skin autofluorescence. Skin autofluorescence was assessed by the AFR (patent number PCT/NL99/00607). In short, the AFR illuminates approximately $1 \mathrm{~cm}^{2}$ of skin (which is guarded against surrounding light) with an excitation light source of 300 to $420 \mathrm{~nm}$ (peak excitation $\sim 350 \mathrm{~nm}$ ). Figure 1 shows a photograph of the AFR and a representative autofluorescence plot. Only light from the skin is measured between 300 and $600 \mathrm{~nm}$ with a spectrometer (PC-1000 fiber optic spectrometer, Ocean Optics; Duiven, The Netherlands) using a 200- $\mu \mathrm{m}$ glass fibre (Farnell, Utrecht, The Netherlands). Autofluorescence was calculated by dividing the average light intensity emitted per $\mathrm{nm}$ over the 420 - to $600-\mathrm{nm}$ range by the average light intensity emitted per nm over the 300- to 420-nm range [24]. Pilot experiments had revealed marked differences in autofluorescence between diabetic and control persons at these excitation and emission ranges. In studies on corneal autofluorescence, comparable ranges have been used or have shown the highest relative excitation efficiency [24, 25, 26].

Because skin pigmentation may absorb light and thus influence autofluorescence, skin reflection measurements across the 300- to 420-nm range were compared with those of a white Teflon block (assuming 100\% reflectance) [27]. Skin reflection was calculated by dividing the mean intensity of light reflected from the skin by the mean intensity reflected from the white Teflon block across the 300 - to 420 -nm range.

All measurements were performed at room temperature in a semi-dark environment. After control measurements had been 
made with the lamp off (dark), autofluorescence of the skin was measured six times (every $10 \mathrm{~s}$ ) at the volar side of the arm, approximately $10 \mathrm{~cm}$ below the elbow fold, and at the dorsal side of the lower leg (calf). Care was taken to perform the measurement at a normal skin site, i.e. one without visible vessels, scars, lichenification, or other skin abnormalities. Repeated AFR measurements taken over a single day in control subjects and diabetic patients showed an overall Altman error percentage of $5.03 \%$. Intra-individual seasonal variance among control subjects and diabetic patients showed an Altman error percentage of $5.87 \%$. The differences between repeated measurements were not dependent on the level of autofluorescence. Autofluorescence was calculated off-line by automated analysis and was observer-independent.

Subjects. We non-invasively measured skin autofluorescence in 46 diabetic patients and 46 control subjects, and obtained skin biopsies from 43 of them. The large majority of subjects (42 of 46 in both groups) were Caucasian. Both Type 1 (age $<30$ years at diagnosis and insulin-dependent) and Type 2 diabetic patients were randomly recruited from our diabetes outpatient clinic, University Hospitz Groningen. In control subjects, diabetes mellitus and renal failure were excluded by conventional criteria (American Diabetes Association) and measurement of serum creatinine levels $(<120 \mu \mathrm{mol} / \mathrm{l})$. Control subjects were age- (within 5 years age range) and sex-matched to diabetic patients.

Duration of diabetes, $\mathrm{HbA}_{1} \mathrm{c}$ closest to the date of autofluorescence measurement, the mean $\mathrm{HbA}_{1} \mathrm{c}$ value of approximately four measurements taken in the previous year, serum creatinine levels and smoking habits were obtained from chart review. The local ethics committee approved this study and informed consent was obtained prior to the measurements being taken.

Skin biopsies. Full-thickness punch skin biopsies (4 mm) were taken from the volar side of the lower arm (at the same location as the AFR measurement) under 2\% lidocaine local anaesthetic. Skin samples were frozen in liquid nitrogen and subsequently stored at $-80{ }^{\circ} \mathrm{C}$. They were later analysed in a single batch for collagen-linked fluorescence (CLF; excitation at $370 \mathrm{~nm}$, emission at $440 \mathrm{~nm}$ ) following pepsin digestion, and for pentosidine (by HPLC), $N^{\varepsilon}$-(carboxyethyl)lysine (CEL) and CML (by gas chromatography and mass spectrometry), as described previously [28, 29].
Briefly, CLF was measured in collagen solubilised by digestion with $2 \%$ pepsin in $0.5-\mathrm{mol} / 1$ acetic acid for $24 \mathrm{~h}$ at $37^{\circ} \mathrm{C}$. Fluorescence was measured using excitation and emission wavelengths of $370 \mathrm{~nm}$ and $440 \mathrm{~nm}$ respectively. Pentosidine was measured in collagen hydrolysates by reversed-phase HPLC using post-column fluorescence detection (excitation at $328 \mathrm{~nm}$, emission at $378 \mathrm{~nm}$ ). Both CLF and pentosidine were normalised to the hydroxyproline content of collagen, measured according to the method of Stegemann and Stalder, as modified by Maekawa et al. [30]. CML and CEL in collagen hydrolysates were measured as their $N$-trifluoroacetyl methyl esters by isotope dilution, selected ion monitoring gas chromatography and mass spectrometry, and were normalised to the lysine content of collagen.

Samples were analysed at random, without knowledge of clinical and AFR characteristics.

Statistical methods. All variables had a normal distribution (Kolmogorov-Smirnov test, $p>0.05$ ). Differences in autofluorescence between diabetic patients and matched controls were analysed using the Student's $t$ test.

Correlation analyses were performed by Pearson correlation. Multivariable stepwise regression analyses were performed to determine independent effects on autofluorescence. For analysis of the relationship between autofluorescence and skin AGE levels in biopsies, only the autofluorescence measurements at the lower arm are used. A two-tailed $p$ value of less than 0.05 was considered statistically significant. Data are shown as means $\pm \mathrm{SD}$.

\section{Results}

Validation of the AFR against skin biopsy fluorescence and skin AGE. The baseline characteristics of the 13 Type 1 diabetic patients, 18 Type 2 diabetic patients and 12 control subjects studied to validate the AFR against specific AGE levels in skin biopsies are shown in Table 1. Figure 2a shows the correlation between skin autofluorescence and CLF $(r=0.62, p<0.001)$. Autofluorescence also correlated with CLF for each of the two separate diabetic subgroups (Type $1 r=0.64$, Type $2 r=0.58 ; p=0.01$ ).

Table 1. Group characteristics of the diabetic patients and control subjects in whom skin biopsies were retrieved for the validation study

\begin{tabular}{|c|c|c|c|}
\hline Characteristic & $\begin{array}{l}\text { Type } 1 \text { diabetic patients } \\
(n=13)\end{array}$ & $\begin{array}{l}\text { Type } 2 \text { diabetic patients } \\
(n=18)\end{array}$ & $\begin{array}{l}\text { Control subjects } \\
(n=12)\end{array}$ \\
\hline Age (years) & $43 \pm 13$ & $58 \pm 13$ & $45 \pm 25$ \\
\hline Smoking $(n)$ & 4 & 2 & 7 \\
\hline Creatinine $(\mu \mathrm{mol} / \mathrm{l})$ & $90 \pm 10$ & $91 \pm 14$ & $80 \pm 13^{b}$ \\
\hline Duration of diabetes (years) & $22 \pm 9$ & $15 \pm 11^{\mathrm{a}}$ & NA \\
\hline Pentosidine (pmol/ $\mu \mathrm{g}$ hydroxyproline) & $0.080 \pm 0.034$ & $0.120 \pm 0.041$ & $0.060 \pm 0.025^{\mathrm{b}}$ \\
\hline CML (mmol/mol lysine) & $0.88 \pm 0.21$ & $1.07 \pm 0.38$ & $0.58 \pm 0.23^{\mathrm{b}}$ \\
\hline CEL (mmol/mol lysine) & $0.122 \pm 0.002$ & $0.128 \pm 0.041$ & $0.086 \pm 0.028^{b}$ \\
\hline Autofluorescence (AU) & $0.016 \pm 0.004$ & $0.018 \pm 0.005$ & $0.013 \pm 0.003^{b}$ \\
\hline Skin reflectance, arm (\%) & $18.5 \pm 10.1$ & $12.1 \pm 4.9^{\mathrm{a}}$ & $18.5 \pm 8.7$ \\
\hline
\end{tabular}

Values are means $\pm \mathrm{SD}$, except when indicated. ${ }^{\mathrm{a}} p<0.05$ vs Type 1 diabetic patients; ${ }^{\mathrm{b}} p<0.05$ vs diabetic patients. CEL, $N^{\varepsilon_{-}}(\mathrm{car}-$ boxyethyl)lysine; CLF, collagen-linked fluorescence; CML, $N^{\varepsilon}$-(carboxymethyl)lysine; NA, not applicable 


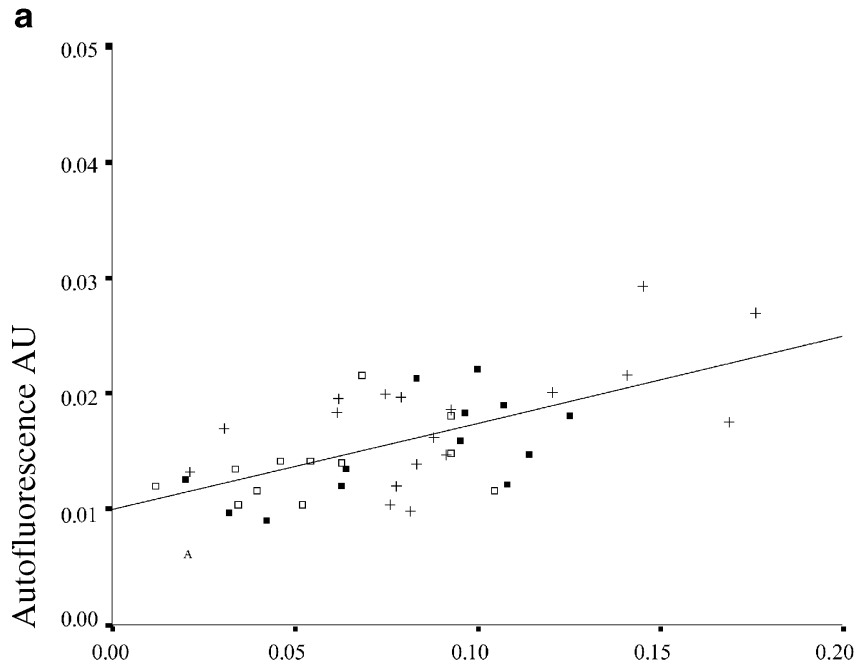

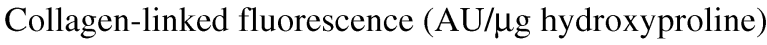

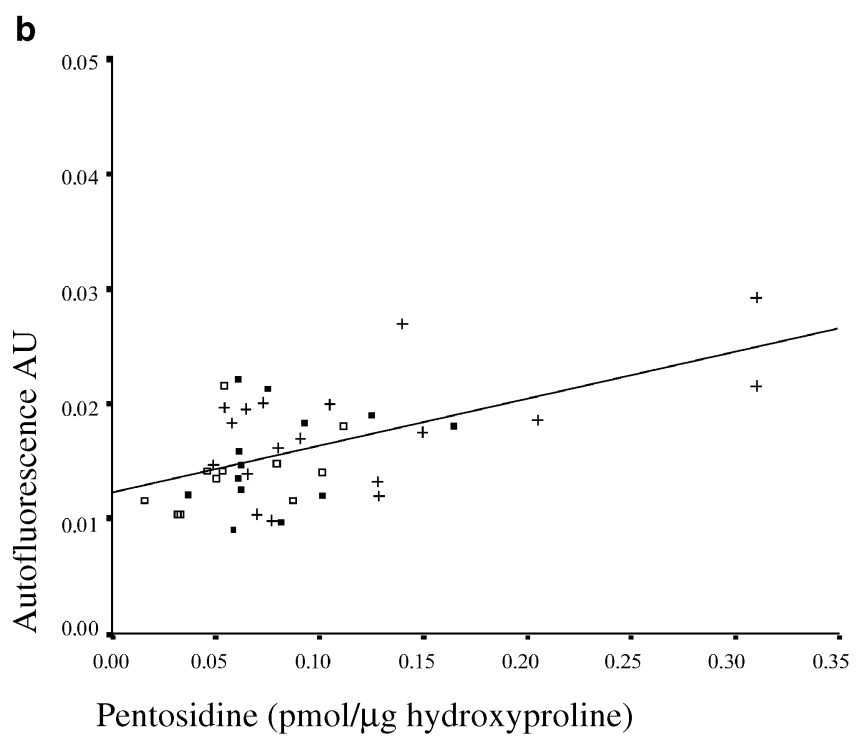

Fig. 2. The relationship between non-invasive skin autofluorescence measurements and (a) skin collagen-linked fluorescence and (b) pentosidine levels in skin biopsies. $\square$ denotes results from Type 1 diabetic patients; + results from Type 2 diabetic patients; $\square$ results from control subjects

Furthermore, autofluorescence correlated with skin levels of pentosidine $(r=0.55, p<0.001$; Fig. $2 \mathrm{~b})$, CML $(r=0.55, p<0.001)$ and CEL $(r=0.47, p=0.002)$. Skin biopsy levels of CLF correlated with levels of pentosidine $(r=0.55, p<0.001)$, CML $(r=0.50, p=0.001)$ and CEL $(r=0.37, p=0.01)$. Correlations between the levels of pentosidine, CML and CEL all had correlation coefficients greater than 0.4 . Skin reflection negatively correlated with pentosidine $(r=-0.39, p=0.01)$, CML $(r=-0.32, p=0.04)$, and CEL $(r=-0.32, p=0.04)$.

Autofluorescence in diabetic patients and control subjects. Table 2 describes the baseline characteristics of the 46 diabetic patients and 46 control subjects studied to analyse the relationship between skin autofluores-

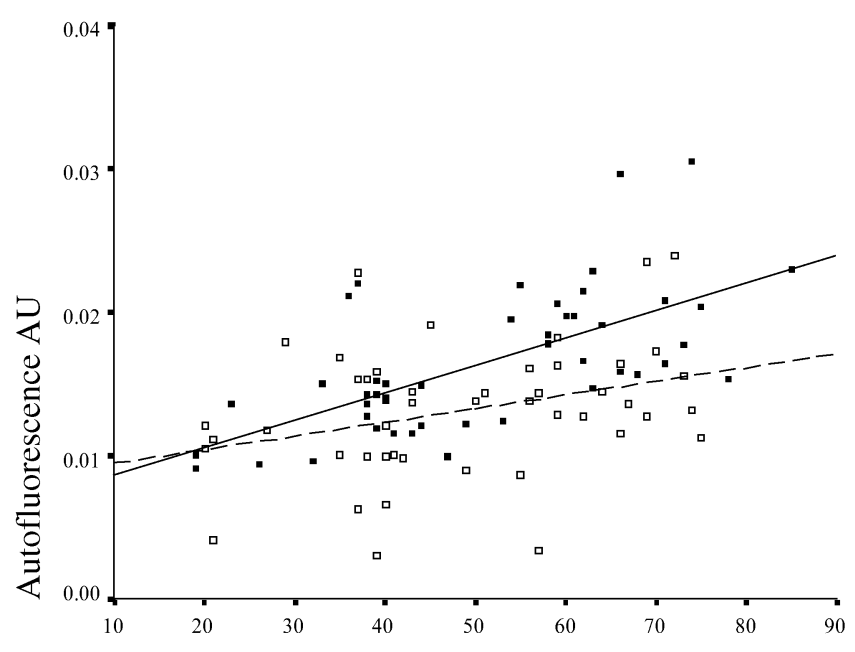

Age (years)

Fig. 3. The relationship between non-invasive skin autofluorescence measurements and age in diabetic patients ( $\boldsymbol{\square}$, continuous line) and control subjects ( $\square$, dotted line)

cence and diabetes mellitus. The amount of light reflected by the skin $(\sim 19 \%)$ did not vary between diabetic patients and control subjects. Figures $1 \mathrm{~b}$ and $1 \mathrm{c}$ show plots of autofluorescence intensity. Autofluorescence measured at the lower arm and lower leg correlated strongly with each other $(r=0.98)$. Mean autofluorescence was $25 \%$ higher in the group of diabetic patients $(0.0164 \pm 0.0047 \mathrm{AU})$ than in the control subjects $(0.0131 \pm 0.0047 \mathrm{AU}, p=0.001)$. Mean autofluorescence was higher in the group of Type 2 diabetic patients $(0.019 \pm 0.0047 \mathrm{AU})$ than in the group of Type 1 diabetic patients $(0.015 \pm 0.005, p=0.002)$, but Type 2 diabetic patients were older and had somewhat higher mean $\mathrm{HbA}_{1} \mathrm{c}$.

Autofluorescence correlated (Table 3) with age, diabetes duration, serum creatinine levels and mean $\mathrm{HbA}_{1} \mathrm{c}$ of the previous year, but not with the $\mathrm{HbA}_{1} \mathrm{c}$ value closest to the date of autofluorescence measurement. The age-related rate of autofluorescence increase was 2.1 times higher in diabetic patients than in control subjects. The overall variance in autofluorescence observed among diabetic patients could largely $(r=0.78, p<0.001)$ be explained by the independent effects of age $(p<0.001)$, mean $\mathrm{HbA}_{1} \mathrm{c}(p<0.001)$ and serum creatinine levels $(p=0.01)$. In control subjects, the overall variance in autofluorescence could be explained by the independent effects of age $(p=0.01)$ and smoking $(p=0.04)(r=0.435, p=0.01)$. Smoking was not related to skin autofluorescence variance in diabetic patients.

Figure 3 shows the relationship between autofluorescence and age in diabetic and control subjects. A total of 32 of the 46 diabetic patients (70\%) had autofluorescence values above the $95 \% \mathrm{CI}$ of the mean relation between autofluorescence and age in control subjects. Of the remaining 14 diabetic patients, nine had a 
Table 2. Characteristics of subjects enrolled in the case-control study comparing the effects of diabetes on autofluorescence

\begin{tabular}{lccc}
\hline Characteristic & Type 1 diabetic patients $(n=25)$ & Type 2 diabetic patients $(n=21)$ & Control subjects $(n=46)$ \\
\hline Age (years) & $43 \pm 16$ & $60 \pm 11^{\mathrm{a}}$ & $50 \pm 16$ \\
Sex (male/female) & $13 / 12$ & $12 / 9$ & $25 / 21$ \\
Smokers $(n)$ & 5 & 3 & $19^{\mathrm{b}}$ \\
Caucasian $(\%)$ & 92 & 95 & 91 \\
Skin reflectance, arm $(\%)$ & $19.1 \pm 0.07$ & $18.1 \pm 0.07$ & $17.7 \pm 6.3$ \\
Creatinine $(\mu$ mol/l) & $91 \pm 12$ & $94 \pm 17$ & $82 \pm 15^{\mathrm{b}}$ \\
Duration of diabetes (years) & $25 \pm 13$ & $16 \pm 12^{\mathrm{a}}$ & NA \\
Mean HbA $(\%)^{\mathrm{c}}$ & $7.5 \pm 1.0$ & $8.3 \pm 1.2^{\mathrm{a}}$ & $\mathrm{NA}$ \\
ACE-inhibitor $(n)^{\mathrm{d}}$ & 8 & 8 & $\mathrm{NA}$ \\
Statins $(n)^{\mathrm{d}}$ & 4 & $12^{\mathrm{a}}$ & $\mathrm{NA}$ \\
Antioxidants $(n)^{\mathrm{d}}$ & 4 & $17^{\mathrm{a}}$ & $\mathrm{NA}$ \\
Autofluorescence $(\mathrm{AU})$ & $0.015 \pm 0.005$ & $0.019 \pm 0.005^{\mathrm{a}}$ & $0.013 \pm 0.005^{\mathrm{b}}$
\end{tabular}

Values are means $\pm \mathrm{SD}$, except when indicated. a $p<0.05 \mathrm{vs}$ Type 1 diabetic patients; ${ }^{\mathrm{b}} p<0.05$ between diabetic patients and control subjects; ${ }^{\mathrm{c}}$ mean of approximately four measure- ments taken in the previous year; d number of patients taking this medication. NA, not applicable

Table 3. Correlations between non-invasive skin autofluorescence measurements and other recorded parameters in diabetic patients and control subjects

\begin{tabular}{llll}
\hline & Type 1 diabetes patients $(n=25)$ & Type 2 diabetes patients $(n=21)$ & Control subjects $(n=46)$ \\
\hline Age $($ years $)$ & $r=0.595(p=0.002)$ & $r=0.479(p=0.028)$ & $r=0.326(p=0.027)$ \\
Duration of diabetes (years) & $r=0.821(p<0.001)$ & $r=0.640(p=0.002)$ & NA \\
Mean $\mathrm{HbA}_{1} \mathrm{c}(\%)^{\mathrm{a}}$ & $r=0.565(p=0.003)$ & $r=0.495(p=0.023)$ & $\mathrm{NA}$ \\
Single $\mathrm{HbA}_{1} \mathrm{c}(\%)^{\mathrm{b}}$ & $r=0.404(p=0.051)$ & $r=0.348(p=0.123)$ & NA \\
Creatinine $(\mu \mathrm{mol} / \mathrm{l})$ & $r=0.578(p=0.002)$ & $r=0.657(p=0.001)$ & $r=0.061(p=0.699)$ \\
\hline
\end{tabular}

a Mean of readings taken over the previous year; ${ }^{b}$ reading taken closest to the date of autofluorescence measurement. NA, not applicable

mean $\mathrm{HbA}_{1} \mathrm{c}$ below $6.8 \%$. Of the ten control subjects with autofluorescence values above their matched diabetic patient, eight were smokers, six of whom had smoked for more than 20 years. Control subjects who smoked were approximately 15 years older than their non-smoking counterparts as defined by the relationship between skin autofluorescence and age.

\section{Discussion}

Our study shows that autofluorescence, measured non-invasively in the skin, is related to skin AGE accumulation in diabetic patients and control subjects. Furthermore, skin autofluorescence measured with the AFR is increased in diabetic patients and seems to be related to risk factors for long-term diabetic complications, such as glycaemic control. The existing overlap between diabetic and control subjects and the strong age dependence of autofluorescence limit its use as a diagnostic test. However, the association between AGE and chronic complications of diabetes mellitus, renal failure and other age-related conditions, and experimental evidence for the pathogenic role of AGE in the development of complications, suggest that the AFR could be a promising tool for risk assessment.
This technique is limited by the fact that not all AGE exhibit fluorescent properties. ELISA-based assays have shown that increased accumulation of specific AGE occurs prior to increased autofluorescence in diabetes [12]. Fluorescence, as expressed in our autofluorescence ratio, is a group reactivity; consequently, it cannot provide quantitative information on the concentrations of individual compounds. Moreover, some non-fluorescent AGE, such as CML, are thought to play an important pathogenic role. Interestingly, in our study, skin autofluorescence correlated with skin levels of non-fluorescent AGE. Thus, skin autofluorescence may be a marker of the behaviour of the total skin AGE pool. The parallel relationships between CLF and both pentosidine (fluorescent) and CML (not fluorescent), and the interrelationships between the specific AGE also suggest that fluorescent and non-fluorescent AGE behave similarly and fluorescence may be used as a marker.

Our study is also limited by the fact that we cannot rule out the possibility that other fluorophores, e.g. $\mathrm{NADH}$, or differences in absorption due, for example, to haemoglobin, may interfere with the relationship between autofluorescence and AGE accumulation [26]. Nevertheless, skin biopsy fluorescence itself is strongly related to long-term diabetic complications $[5,8]$. 
Exposure to sunlight as a source of skin photo-ageing is a possible confounding factor in skin AGE accumulation, but the similarity between AFR measurements taken at the lower arm and the calf sites suggests that this effect is probably limited. The vast majority of subjects included in the present study were Caucasian patients; hence, the results cannot address the issue of racial differences. Although corrections using differences in skin reflectance may resolve the confounding effects of skin pigmentation on skin autofluorescence, and seasonal intra-individual variance shows a low Altman error percentage, our conclusions on the value of autofluorescence as a marker of AGE accumulation are for the present limited to non-pigmented skin.

Although mean skin reflection was not significantly different between the groups of diabetic patients and control subjects, skin reflection correlated negatively with specific AGE accumulation. This observation may be related to the characteristic brown-yellow adducts that AGE form.

The correlation we found, both in diabetic patients and in control subjects, between skin autofluorescence measured non-invasively and age is similar to previously observed correlations between CLF and age [5, 30]. The 2.1-times higher age-related increase in autofluorescence in diabetic patients compared with in control subjects is of a similar magnitude to that reported by Monnier et al. [5] for CLF (2.4 times higher). This increase may be partly explained by the relationship we found between autofluorescence and diabetes duration, serum creatinine levels and mean $\mathrm{HbA}_{1} \mathrm{c}$ values of the previous year. Mean $\mathrm{HbA}_{1} \mathrm{c}$ levels in diabetic patients were also significantly, but not strongly, related to (advanced) glycated collagen levels in the DCCT study [8]. As in our study, Monnier et al. [5] found that the relationship between levels of (advanced) glycated collagen and the mean $\mathrm{HbA}_{1} \mathrm{c}$ over the year preceding the skin biopsy was stronger than that between (advanced) glycated collagen and the $\mathrm{HbA}_{1} \mathrm{c}$ value measured nearest the biopsy. Skin autofluorescence may provide a longer-term index of tissue damage related to metabolic stress than shorter-term markers, such as glycated haemoglobin. Wolffenbuttel et al. [31] observed a slower decline in haemoglobin-AGE, the AGE-modified form of haemoglobin, than in $\mathrm{HbA}_{1} \mathrm{c}$ in diabetic patients switching to a more intensive regimen. AGE skin levels increase in parallel with the accumulation of AGE in other organs [5] and correlate with early manifestations of nephropathy and retinopathy independently of $\mathrm{HbA}_{1} \mathrm{c}[8,32,33]$.

Importantly, AGE accumulation is not only a measure of traditional risk factors for long-term diabetic complications (e.g. glycaemic control), but also of oxidative stress status and renal function. In our study, creatinine levels were independently related to skin autofluorescence in diabetic patients. Renal dysfunc- tion in diabetic patients, but also in euglycaemic patients, contributes to increased tissue AGE accumulation and to the development of cardiovascular complications in these patients $[3,12,13]$. Smoking, which is recognised as a source of oxidative stress in vivo [32], was also correlated with increased skin autofluorescence in control subjects. The majority of the ten control subjects with higher autofluorescence values than their matched diabetic patient had smoked for many years. Tobacco smoke contains fluorescent cross-linking proteins and increases plasma AGE [32]. In diabetic patients, skin autofluorescence was not related to smoking, possibly due to the low number of smokers in the diabetic population. Furthermore, the metabolic consequences of diabetes may have a higher impact on AGE accumulation than oxidative stress induced by smoking. Tissue AGE accumulation may be further influenced by nutrition [34].

As discussed above, the AFR is not intended as a screening technique for diagnosing diabetes mellitus. Its value may instead lie in the risk assessment of AGE-related complications. The current study was too small, and also not intended to address the relationship between autofluorescence and existing or new complications, nor was it designed to address possible differences in age-related increases in autofluorescence between Type 1 and Type 2 diabetic patients.

The efficacy of the AFR for predicting progression of diabetic complications and mortality is now being investigated in a large (>1000 Type 2 diabetic patients) prospective cohort study. Apart from plasma AGE and haemoglobin-AGE assays, few tools are currently available for monitoring the effects of interventions aimed at reducing AGE accumulation, such as aminoguanidine or AGE-breakers [16, 17, 18]. The AFR could be a promising alternative for monitoring interventions for the following reasons: (i) disparate changes between plasma and tissue AGE levels may occur [19], and tissue AGE accumulation may be more relevant to the development of tissue damage than plasma levels; (ii) current plasma AGE assays are time-consuming and the results can be difficult to reproduce or standardise between laboratories.

In conclusion, our results show that the AFR measures AGE accumulation non-invasively in non-pigmented skin. The accumulation of AGE is thought to mediate the deleterious effects of hyperglycaemia and oxidative stress in conditions such as diabetes and renal failure. Consequently, simple and rapid noninvasive measurement of skin autofluorescence could be a convenient way of assessing both the risk of related complications and the effects of interventions aimed at reducing AGE.

Acknowledgements. This work was supported by grants from the USA National Institutes of Diabetes, Digestive and Kidney Diseases (DK-19971), and from the Dutch Diabetes Fund (DFN 2000.00.006). We would like to express our gratitude to W. J. Sluiter for reviewing this article. 


\section{References}

1. Schleicher E, Wagner E, Nerlich AG (1997) Increased accumulation of the glycoxidation product $N^{\varepsilon}$-(carboxymethyl)lysine in human tissues in diabetes and aging. J Clin Invest 99:457-468

2. Stitt AW, He C, Friedman S et al. (1997) Elevated AGEmodified ApoB in sera of euglycemic, normolipidemic patients with atherosclerosis: relationship to tissue AGEs. Mol Med 3:617-627

3. Miyata T, Wada Y, Cai Z et al. (1997) Implication of an increased oxidative stress in the formation of advanced glycation end products in patients with end-stage renal failure. Kidney Int 51:1170-1181

4. Smith MA, Taneda S, Richey PL et al. (1994) Advanced Maillard reaction end products are associated with Alzheimer disease pathology. Proc Natl Acad Sci USA 91:5710-5714

5. Monnier VM, Vishwanath V, Frank KE, Elmets CA, Dauchot P, Kohn RR (1986) Relation between complications of type 1 diabetes mellitus and collagen-linked fluorescence. N Eng J Med 314:403-408

6. Baynes JW, Thorpe SR (1999) Role of oxidative stress in diabetic complications. A new perspective on an old Paradigm. Diabetes 48:1-9

7. The DCCT research group (1993) The effect of intensive diabetes treatment on the development and progression of long-term complications in insulin-dependent diabetes: The Diabetes Control and Complications Trial. N Engl J Med 329:977-986

8. Monnier VM, Bautista O, Kenny D et al. (1999) Skin collagen glycation, glycoxidation, and crosslinking are lower in subjects with long-term intensive versus conventional therapy of type 1 diabetes. Diabetes 48:870-880

9. Monnier VM, Sell DR, Abdul-Karim FW, Emancipator SN (1988) Collagen browning and cross-linking are increased in chronic experimental hyperglycemia. Relevance to diabetes and ageing. Diabetes 37:867-872

10. Nishikawa T, Edelstein D, Du XL et al. (2000) Normalizing mitochondrial superoxide production blocks three pathways of hyperglycaemic damage. Nature 404:787-790

11. Thornalley PJ (1998) Cell activation by glycated proteins, AGE receptors, receptor recognition factors and functional classification of AGE. Cell Mol Biol 44:1013-1023

12. Beisswenger PJ, Makita Z, Curphey TJ et al. (1995) Formation of immunochemical advanced glycosylation end products precede and correlate with early manifestations of renal and retinal disease in diabetes. Diabetes 44:824-829

13. Makita Z, Radoff S, Rayfield EJ et al. (1991) Advanced glycosylation end products in patients with diabetic nephropathy. N Engl J Med 325:836-842

14. Hammes HP, Brownlee M, Lin J, Schleicher E, Bretzel RG (1999) Diabetic retinopathy risk correlates with intracellular concentrations of the glycoxidation product $\mathrm{N}$-epsilon-(carboxymethyl) lysine independently of glycohaemoglobin concentrations. Diabetologia 42:603-607

15. Kiuchi K, Nejima J, Takano T, Ohta M, Hashimoto $H$ (2001) Increased serum concentrations of advanced glycation end products: a marker of coronary artery disease activity in type 2 diabetic patients. Heart 85:87-91

16. Nilsson BO (1999) Biological effects of aminoguanidine: an update. Inflamm Res 48:509-515

17. Wolffenbuttel BH, Boulanger CM, Crijns FR et al. (1998) Breakers of advanced glycation end products restore large artery properties in experimental diabetes. Proc Natl Acad Sci USA 95:4630-4634
18. Soulis-Liparota T, Cooper M, Papazoglou D, Clarke B, Jerums G (1991) Retardation by aminoguanidine of development of albuminuria, mesangial expansion, and tissue fluorescence in streptozotocin-induced diabetic rats. Diabetes 40:1328-1335

19. Hricik DE, Wu YC, Schulak A, Friedlander MA (1996) Disparate changes in plasma and tissue pentosidine levels after kidney and kidney-pancreas transplantation. Clin Transplantation 10:568-573

20. Dorrian CA, Cathcart S, Clausen J, Shapiro D, Dominiczak MH (1998) Factors in human serum interfere with the measurement of advanced glycation endproducts. Cell Mol Biol 44:1069-1079

21. Jager J, Oomen PHN, Sluiter WJ, Reitsma WD, Smit AJ (1997) Improved reproducibility of the "Large-Window" method of assessing transcapillary and interstitial fluorescein diffusion in the skin in healthy subjects and in subjects with insulin-dependent diabetes mellitus. Int J Microcirc Clin Exp 17:150-158

22. Sato E, Mori F, Igarashi S et al. (2001) Corneal advanced glycation end products increase in patients with proliferative diabetic retinopathy. Diabetes Care 24:479-482

23. Abiko T, Abiko A, Ishiko S et al. (1999) Relationship between autofluorescence and advanced glycation end products in diabetic lenses. Exp Eye Res 68:361-366

24. Ishino Y, Yokoi N, Yashara T et al. (2001) Investigation of corneal autofluorescence in diabetic patients. Jpn J Opthalmol 45:116

25. Van Schaik HJ, Alkemade C, Swart W, van Best JA (1999) Autofluorescence of the diabetic and healthy human cornea in vivo at different excitation wavelengths. Exp Eye Res 68:1-8

26. Coremans JMC, Ince C, Bruining HA, Puppels GJ (1997) (Semi-) quantitative analysis of reduced nicotinamide adenine dinucleotide fluorescence images of blood perfused rat heart. Biophys J 72:1849-1860

27. Na R, Stender IM, Henriksen M, Wulf HC (2001) Autofluorescence of human skin is age-related after correction for skin pigmentation and redness. J Invest Dermatol 116:536-540

28. Dyer DG, Dunn JA, Thorpe SR et al. (1993) Accumulation of Maillard reaction products in skin collagen in diabetes and aging. J Clin Invest 91:2463-2469

29. Ahmed MU, Brinkmann-Frye E, Degenhardt TP, Thorpe SR, Baynes JW (1997) $N^{\varepsilon}$-(carboxymethyl)lysine, a product of chemical modification of protein by methylglyoxal, increases with age in human lens proteins. Biochem $\mathrm{J}$ 324:565-570

30. Maekawa T, Rathinasamy TK, Altman KI, Forbes WF (1970) Changes in collagen with age. 1. The extraction of acid soluble collagen from the skin of mice. Exp Gerontol 5:177-186

31. Wolffenbuttel BHR, Giordano D, Founds HW, Bucala R (1996) Long-term assessment of glucose control by haemoglobin-AGE measurement. Lancet 347:513-515

32. Nicoll ID, Stitt AW, Moore JE (1998) Increased levels of advanced glycation endproducts in the lenses and blood vessels of cigarette smokers. Mol Med 4:594-601

33. McCance DR, Dyer DG, Dunn JA et al. (1993) Maillard reaction products and their relation to complications in insulin-dependent diabetes mellitus. J Clin Invest 91:24702478

34. Koschinsky T, He CJ, Mitsuhashi T et al. (1997) Orally absorbed reactive glycation products (glycotoxins): an environmental risk factor in diabetic nephropathy. Proc Natl Acad Sci USA 94:6474-6479 